\title{
AXMACHER, Elke, Johann Arndt und Paul Gerhardt. Studien zur Theologie, Frömmigkeit und geistlichen Dichtung des 17. Jahrhunderts
}

Patrice Veit

\section{OpenEdition}

\section{Journals}

Édition électronique

URL : http://journals.openedition.org/ifha/1110

DOI : 10.4000/ifha. 1110

ISSN : 2198-8943

\section{Éditeur}

IFRA - Institut franco-allemand (sciences historiques et sociales)

Référence électronique

Patrice Veit, «AXMACHER, Elke, Johann Arndt und Paul Gerhardt. Studien zur Theologie, Frömmigkeit und geistlichen Dichtung des 17. Jahrhunderts », Revue de I'IFHA [En ligne], Date de recension, mis en ligne le 01 janvier 2003, consulté le 22 septembre 2020. URL : http://journals.openedition.org/ifha/1110 ; DOI : https://doi.org/10.4000/ifha.1110

Ce document a été généré automatiquement le 22 septembre 2020.

(CIFHA 


\title{
AXMACHER, Elke, Johann Arndt und Paul Gerhardt. Studien zur Theologie, Frömmigkeit und geistlichen Dichtung des 17. Jahrhunderts
}

\author{
Patrice Veit
}

Publié dans la collection du groupe de recherches interdisciplinaires sur les recueils de cantiques de l'université de Mayence, l'ouvrage d'E.A., théologienne à l'université de Bielefeld et spécialiste de la spiritualité luthérienne au XVIIe s., met en relation deux des personnalités les plus marquantes du luthéranisme du XVIIe s. : le théologien Johann Arndt (1555-1621), auteur de deux « bestsellers » de la littérature de dévotion protestante, le Wahres Christentum et le Paradiesgärtlein, d'une part, et le pasteur et poète berlinois Paul Gerhardt (1607-1676), auteur de quelque 140 cantiques dont certains constituent des piliers de la culture luthérienne, d'autre part. Un certain nombre de choses les séparent a priori : l'expérience d'abord de la guerre de Trente Ans qu'a connue Gerhardt, mais pas Arndt, mais aussi leur place dans le luthéranisme de l'époque : si Arndt fut considéré par les uns comme un mystique et un spiritualiste, prisé par d'autres comme un des rénovateurs de la doctrine luthérienne au XVIIe s., préparant la voie au piétisme, Gerhardt resta sa vie durant un adepte de la stricte orthodoxie luthérienne telle que la prônait la faculté de Wittenberg. Pourtant, l'œuvre hymnique de Paul Gerhardt ne peut être véritablement comprise sans le recours à Johann Arndt ; elle constitue un témoignage important de l'influence des idées réformatrices d'Arndt en matière de spiritualité luthérienne, comme le prouvent en particulier les six cantiques de Gerhardt directement composés à partir de prières du Paradiesgärtlein. L'intention d'E.A. est de soumettre les textes d'Arndt et de Gerhardt, dans la diversité de leurs genres, à une lecture proprement théologique, la littérature de dévotion et les cantiques du XVIIe s. relevant aussi, selon elle, d'une interprétation théologique au sens strict du terme. Il s'agit donc de mettre en évidence la façon dont sont véhiculées la théologie et la doctrine luthériennes par le biais de l'expression chantée et de la méditation, ainsi que les moyens poétiques employés (l'auteur utilise 
en ce sens l'expression de " Frömmigkeitstheologie »), et de dégager plus largement la pluralité des "comportements " théologiques dans le luthéranisme du XVIIe s. L'ouvrage rassemble différents textes qui s'orientent autour de trois grands axes. L'œuvre de Johann Arndt d'abord, en s'intéressant, d'une part, à l'anthropologie qui y est incluse et au rapport dialectique entre doctrine et vie et en s'attachant, d'autre part, aux prières consacrées à la Passion du Christ dans le Paradiesgärtlein. Paul Gerhardt, ensuite, vu sous l'angle du théologien protestant à partir de l'analyse détaillée de six cantiques emblématiques, comme le chant sur la providence divine Befiehl du deine Wege ou la contemplation des plaies du Christ sur la croix O Haupt voll Blut und Wunden. Paul Gerhardt et Johann Arndt et leurs rapports enfin, considérés à partir de la doctrine de l'amitié spirituelle dans la tradition chrétienne et protestante telle qu'elle apparaît dans le Paradiesgärtlein (la prière Um Christliche beständige Freundschafft) et chez Paul Gerhardt (le cantique Jesu, allerliebster Bruder). Un ouvrage donc centré essentiellement sur les textes soumis à une approche et à une analyse théologiques exigeantes qui font ressortir la richesse de la spiritualité luthérienne au XVIIe s., mais pour laquelle on aimerait toutefois que soient également pris en considération les différents autres contextes. 